\title{
Case Report \\ A Case of Paraclinoid Internal Carotid Artery Aneurysm That Suffered Vessel Perforation during Pipeline Flex Placement
}

Hidehisa Nishi, ${ }^{1}$ Akira Ishii, ${ }^{1}$ Masahiko Itani, ${ }^{1,2}$ Takayuki Kikuchi, ${ }^{1}$ Yohei Takenobu, ${ }^{1}$ Yukihiro Yamao, ${ }^{1}$ Hiroyuki Ikeda,${ }^{1}$ Yu Abekura, ${ }^{1}$ and Susumu Miyamoto ${ }^{1}$

Objective: A case that suffered vessel perforation during flow diverter stent placement is reported.

Case Presentation: The patient was a 65-year-old woman with an unruptured aneurysm 10 mm in maximum diameter at the paraclinoid portion of the left internal carotid artery. She developed disturbance of consciousness a few hours after elective flow diverter stent placement, and diffuse subarachnoid hemorrhage was noted on head CT. Although rupture of aneurysm was suspected, bleeding from the left angular artery was confirmed by emergent angiography. Trapping of the same site was carried out. Vessel perforation due to jump-up of the delivery wire was retrospectively estimated to be the cause of bleeding.

Conclusion: Vessel perforation is a serious complication of intracranial endovascular treatment, and its cause and treatment are discussed with a review of the literature.

Keywords > complication, flow diverter, vessel perforation, Pipeline Flex, cerebral aneurysm

\section{Introduction}

In intracranial endovascular treatment, perforation of a normal blood vessel at a site distant from the lesion is rare, with an incidence of only $0.31 \%-0.87 \%,{ }^{1-3)}$ but it is an intraprocedural complication with a very poor prognosis. We report a case of perforation of a distal vessel during flow diverter stent placement for aneurysm of the paraclinoid internal carotid artery caused by the delivery wire of Pipeline Flex (Medtronic, Minneapolis, MN, USA).

\footnotetext{
${ }^{1}$ Department of Neurosurgery, Kyoto University Hospital, Kyoto, Kyoto, Japan

${ }^{2}$ Department of Neurosurgery, Shizuoka General Hospital, Shizuoka, Shizuoka, Japan
}

Received: November 20, 2017; Accepted: May 7, 2018

Corresponding author: Hidehisa Nishi. Department of Neurosurgery, Kyoto University Hospital, 54 Kawaharacho, Shogoin, Sakyo-ku, Kyoto, Kyoto 606-8507, Japan

Email: venturahighway83@gmail.com

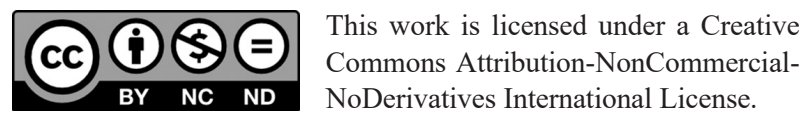

(C)2018 The Japanese Society for Neuroendovascular Therapy

\section{Case Presentation}

The patient was a 65 -year-old woman with a history of hypertension. As unruptured aneurysm was detected in the paraclinoid portion of the left internal carotid artery by head MRI performed at a local hospital for close examination of dizziness, she was referred to our department.

On admission, neurologic examinations showed lucidity, no motor or sensory impairment of the face or limbs, and impairment of the lower visual field of the left eye. On head MRI, the aneurysm was located inside the dura mater, and the left optic nerve was displaced. Cerebral angiography showed that the aneurysm had a neck in the C2-3 segment of the left internal carotid artery, was $10.8 \mathrm{~mm}$ in maximum diameter, $7.7 \mathrm{~mm}$ in height, and $7.3 \mathrm{~mm}$ in neck, and had bleb. In addition, a saccular aneurysm with a maximum diameter of $3 \mathrm{~mm}$ was also detected in the junction of the internal carotid and ophthalmic arteries (Fig. 1).

Since the lesion was symptomatic with visual field deficit and had a maximum diameter exceeding $10 \mathrm{~mm}$, it was judged to be an indication for surgical treatment. We selected flow diverter stent placement because aneurysmal neck clipping would require angioplastic technique in addition to 

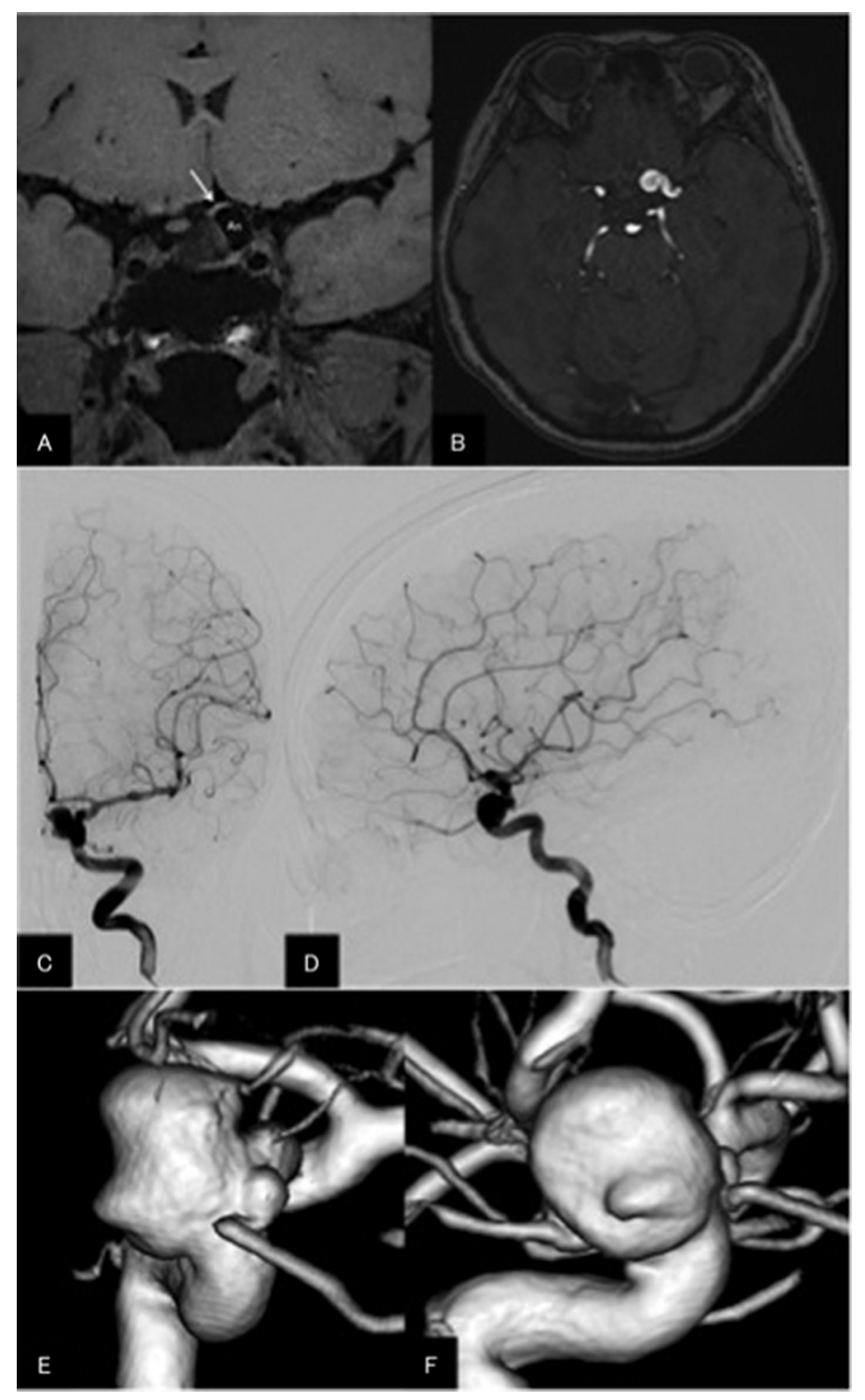

Fig. 1 (A and B) Coronal T1 black-blood image and axial MRA image. Aneurysm dome pushes upward the left optic nerve (arrow). (C and D) Angiograms obtained before the treatment. ( $E$ and $F$ ) 3D rotational angiography shows large paraclinoid aneurysm with bleb.

removal of the anterior clinoid process, and release of the distal dural ring, and because coil embolization would involve the risk of recurrence of aneurysm and impairment of visual function due to mass effect of the coils. Although we considered a combination with coiling for the prevention of delayed aneurysm rupture, we did not take this option since the parent artery was markedly tortuous, and concomitant coil embolization was expected to involve technical difficulties.

Oral dual antiplatelet therapy (aspirin $100 \mathrm{mg}$, clopidogrel $75 \mathrm{mg}$ ) was initiated 2 weeks before the procedure, the platelet aggregation test was performed using the VerifyNow System (Accumetric Inc., San Diego, CA, USA) on the day before the procedure, and both aspirin resistance unit (Aspirin Reaction Units [ARU]; 391) and platelet reactivity unit (P2Y12 Reaction Units [PRU]; 120) were confirmed to be in well-controlled range.

Endovascular treatment was performed under general anesthesia. An 8 Fr guiding catheter (Roadmaster; Goodman, Aichi, Japan) was placed in the left common carotid artery. In the guiding catheter, Navien 058 (Medtronic) + Marksman (Medtronic) + Traxcess (MicroVention TERUMO, Tustin, CA, USA) were advanced, and Marksman was navigated to the middle cerebral artery distal to the lesion. According to the measurement by preprocedural 


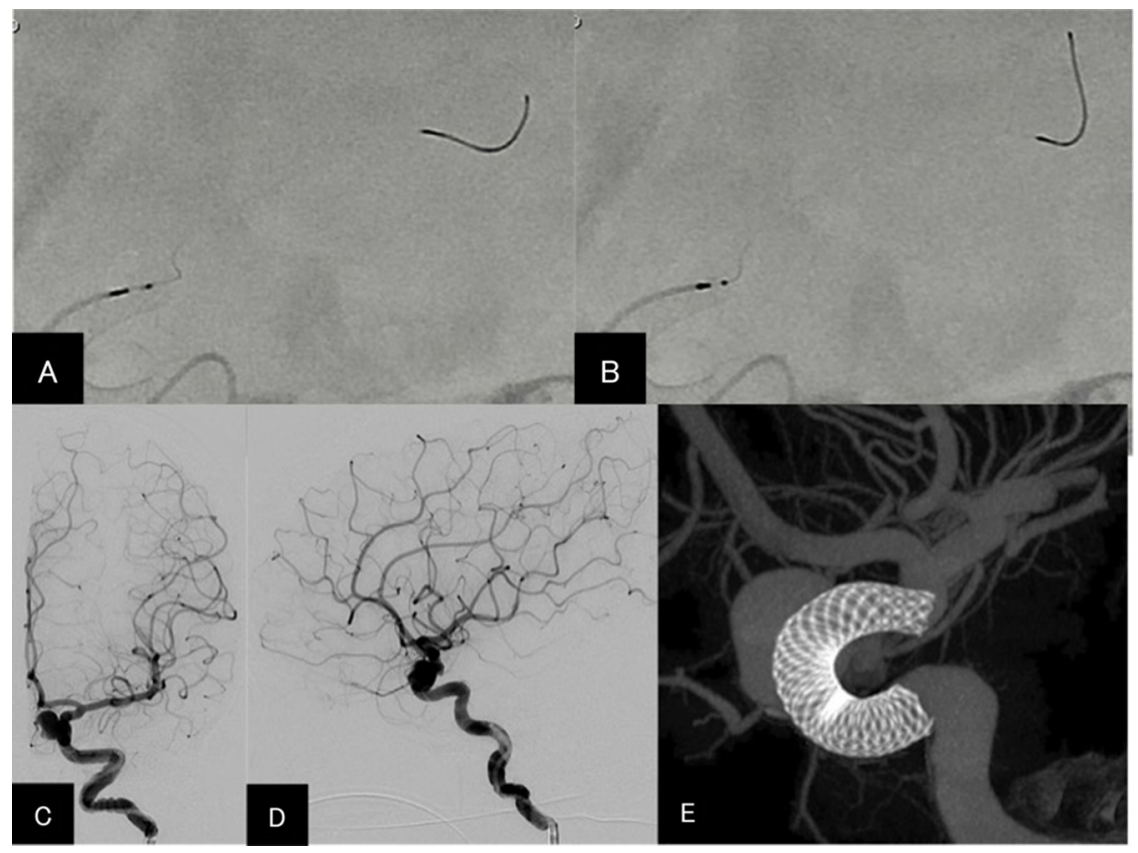

Fig. 2 (A and B) Delivery wire of Pipeline Flex is unintentionally navigated to the distal MCA and jump-up occurred. ( $C$ and $\mathbf{D}$ ) There is no apparent extravasation on the final angiograms of the initial treatment session. (E) Pipeline Flex covers the aneurysm neck and well opposed to the vessel wall on cone beam CT. MCA: middle cerebral artery

three-dimensional rotational angiography (3D-RA), the parent artery was 3.3-4.2 $\mathrm{mm}$ in diameter, and the neck was $7.3 \mathrm{~mm}$ long, so we selected Pipeline Flex $4.25 \times 20 \mathrm{~mm}$. The left internal carotid artery was markedly tortuous at some places from the cervical to intracranial segments, and strong resistance was felt during navigation of Pipeline Flex, but we could manage to guide it into the cranium. After the stent was expanded partially in the M1 segment of the middle cerebral artery, we attempted to reverse the polytetrafluoroethylene (PTFE) sleeve by re-sheathing, but it was impossible due to strong resistance. The stent was deployed from $\mathrm{C} 1$ to $\mathrm{C} 4$ segments of the left internal carotid artery under catheter control. The stent could be placed at the intended site without twisting or flattening (Fig. 2E). Since malapposition was noted on the proximal side of the stent on internal carotid artery angiography after stenting, we attempted to retrieve the delivery wire into the Marksman for balloon dilatation, but it was difficult due to strong resistance. During retrieval of the delivery wire, it was unintendedly advanced to a distal vessel (M2 segment of the middle cerebral artery), where it jumped up further, but it could be retrieved thereafter (Fig. 2A and 2B). After retrieval of the delivery wire, a Transform SC $4 \times 7 \mathrm{~mm}$ (Stryker, Kalamazoo, MI, USA) was guided into the stent, balloon angioplasty was performed in the part of slight malapposition, and the procedure was ended after confirming adequate stent apposition by cone-beam CT. The eclipse sign was not observed in the aneurysm on angiography at the end of the treatment, but as no clear extravasation or filling defect was noted, the treatment was judged at this point to have been successful (Fig. 2C and 2D).

Immediately after the procedure, the patient was awake and alert, and no headache or neurologic deficit was noted. However, as headache appeared 1 hour after the procedure and was accompanied by disturbance of consciousness and vomiting from 3 hours after the procedure, head CT was performed. A large high-density area was noted from the basal cistern to the bilateral sylvian fissures, indicating subarachnoid hemorrhage and acute subdural hematoma (Fig. 3). Since rupture of intracranial aneurysm was suspected, general anesthesia was reinduced, and preparations for emergency intracranial endovascular treatment were made. However, left internal carotid artery angiography showed no bleeding from the aneurysm, and slight extravasation and pooling of the contrast agent were noted in the left angular artery from the capillary to the venous phase (Fig. 4A-4E). We judged this site to be the source of bleeding and decided to perform trapping. The distal and proximal sides of the bleeding site were occluded with coils (ED coil 10 ExtraSoft; Kaneka Medix Corp., Osaka, Japan). Since slight blood flow to the trapped area persisted even after occlusion, 


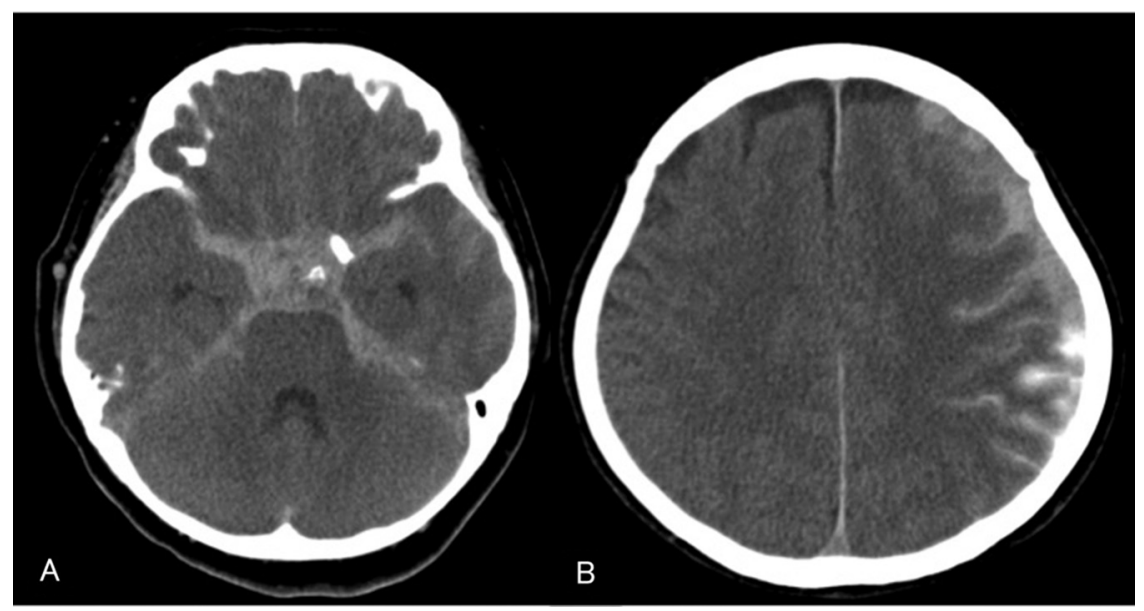

Fig. 3 Post-operative CT shows diffuse subarachnoid hemorrhage (A) and left acute subdural hematoma (B).

the procedure was ended following infusion of $20 \%$ n-butyl-2-cyanoacrylate (NBCA) (Fig. 4F).

After the procedure, delay of word recall, mild motor paralysis of the right upper extremity (Manual Muscle Test [MMT] 4/5), and right hemispatial neglect were observed, and, on MRI, an area of infarction appeared in the left angular artery territory. In addition, an acute symptomatic seizure was observed once. Although dual antiplatelet therapy was continued after the procedure, there was no recanalization of the site of perforation, and no formation of pseudoaneurysm or re-bleeding was noted. However, symptomatic cerebral vasospasm occurred during the course, and intra-arterial infusion of fasudil hydrochloride was performed twice (days 8 and 10). Rehabilitation was continued, eventually resulting in alleviation of motor paralysis, but mild aphasia (delay of word recall) persisted, and the patient was discharged to home with a modified Rankin Scale score of 1 .

\section{Discussion}

\section{Manipulation estimated to have caused bleeding}

From the findings on left internal carotid artery angiography at emergency treatment, the cause of bleeding in this patient was judged to be perforation of the branch of the left middle cerebral artery to angular gyrus (angular artery) (Fig. 4). Retrospective evaluation of fluoroscopic images at treatment suggested that, during retrieval of the delivery wire of Pipeline Flex, the delivery wire jumped up and instantaneously entered the branch of the left middle cerebral artery (Fig. 2). However, roadmap was not used during this maneuver, and we could not confirm whether or not the delivery wire passed out of the vessel through the branch at the jump-up. Since this was the only time when the maneuver involved the left angular artery, and since there is a previous report that jump-up is a risk of vessel perforation, ${ }^{2)}$ jump-up of the delivery wire was estimated to be the cause of vessel perforation in this patient.

In this patient, the cause of jump-up of the delivery wire was speculated as follows: Due to the tortuousness of the approach route, friction of the entire system was increased, external force acting on Marksman (force toward the direction of catheter advancement in the vessel) accumulated in the vessel without our awareness, and the force that accumulated in the system was released on retrieval of the delivery wire after the placement of Pipeline and caused jump-up of the system. Lin et al. reported, concerning their 83 patients who underwent flow diverter stent placement, that, when tortuosity of the parent artery (internal carotid artery) was classified into five types, the procedural time was prolonged, and salvage procedures were performed more frequently (device retrieval or balloon dilatation was needed more often) with increasing tortuosity, suggesting that tortuosity of the parent artery is correlated with the procedural risk in flow diverter stent placement. $\left.{ }^{4}\right)$

\section{Review of the literature concerning perforation of normal vessels in intracranial endovascular treatment}

The incidence of perforation of normal vessels at sites other than the lesion in intracranial endovascular treatment has been reported to be $0.31 \%-0.87 \%{ }^{1-3)}$ (about $5 \%$ for perforation of feeding arteries in embolization of intracranial arteriovenous malformations, ${ }^{5)} 0.5 \%$ and $0.9 \%-7 \%$ for perforation of unruptured and ruptured intracranial 


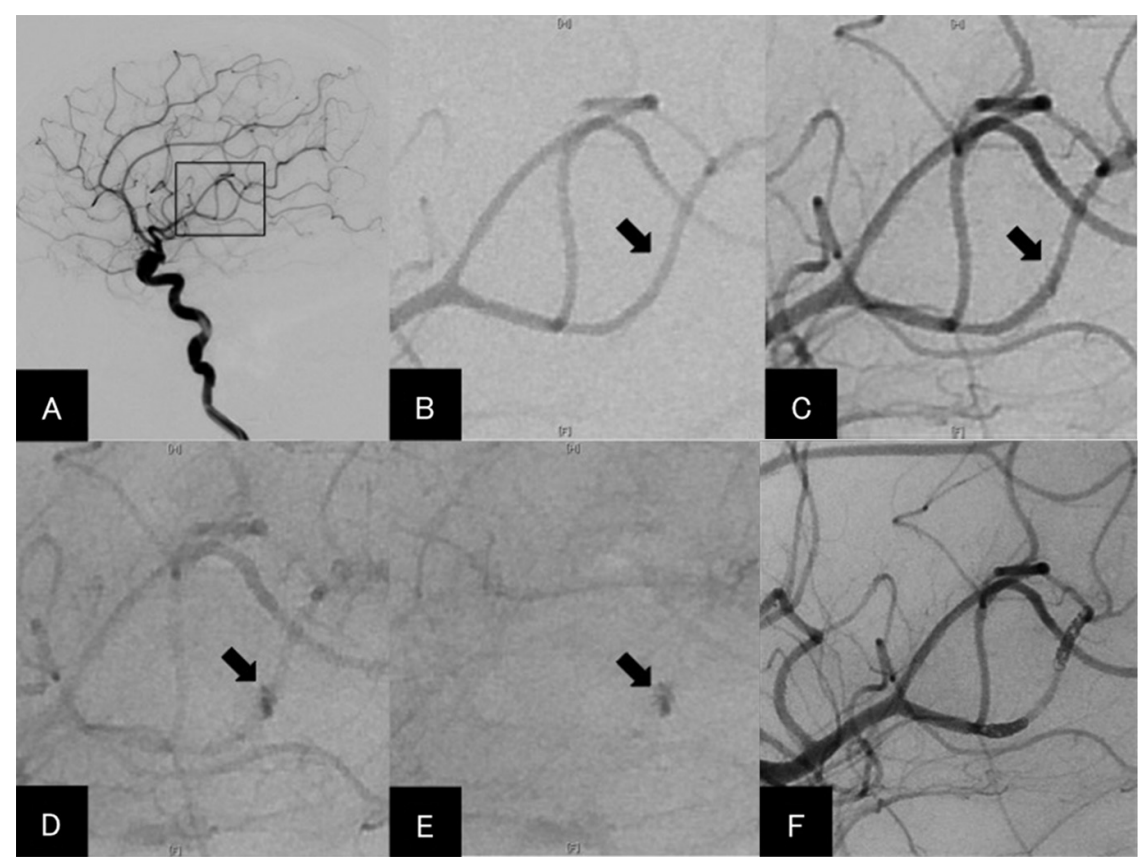

Fig. 4 (A) Angiogram (lateral view). A portion surrounded by a square is magnified in B, C, D, and E. (B-E) Angiograms (lateral view) displayed by time-course. Minor extravasation of left angular artery and pooling of the contrast medium is detected at capillary phase to venous phase (arrows). (F) Left angular artery is trapped by coils and NBCA. NBCA: n-butyl-2-cyanoacrylate

aneurysms, respectively, in coil embolization, ${ }^{2,6-8)}$ and about $4 \%$ for perforation of occluded vessels during mechanical thrombectomy for acute major artery occlusion $^{9,10)}$ ). The incidence of perforation of normal vessels in flow diverter stent placement, a recently introduced technique, has been reported to be about $0.9 \%-9.0 \%$, and the procedure may involve a greater risk of perforation of normal vessels than conventional techniques. ${ }^{11-15)}$

According to our review, there have been 18 cases of iatrogenic perforation of normal intracranial vessels (when the search was limited to the literature providing information including the disease state, cause, treatments, and outcome) $)^{1-3,16-19)}$ (Table 1). Perforation caused subarachnoid hemorrhage in most cases but intracerebral or intraventricular hemorrhage in some (Cases 6-10). In our patient, subarachnoid hemorrhage was accompanied by acute subdural hematoma, perhaps because perforation occurred in a vessel near the brain surface. Also, as symptoms caused by vessel perforation, headache, convulsion, and local brain symptoms that vary with the site of damage, occasionally accompanied by hydrocephalus, have been reported. Our patient also showed convulsion, local brain symptoms, and delayed cerebral vasospasm. The clinical prognosis was very poor, with death of 6 of the 18 cases (mortality: 33\%).

The cause of vessel perforation was direct damage of the vascular wall by the device (catheter or wire) in all previous reports. Anatomical characteristics of blood vessels to be noted in connection with vessel perforation include 1) sharp bifurcations, 2) marked tortuosity, and 3) a small diameter. ${ }^{1,3,16}$ Also, risk factors related to the procedure have been reported to include 1) the use of a rigid guide wire or catheter, 2) no use of roadmap, 3) aggressive catheter manipulation, 4) jump-up due to exchange technique, and 5) use of a guide wire with a slit at the end (e.g., Synchro2 guidewire). ${ }^{1,3,19)}$ In addition to these risk factors, increased friction of the entire system due to tortuousness of the approach route as observed in our patients, which markedly impairs the operability and may cause unintended movement of the device, is also considered to require attention. Puffer et al. ${ }^{15)}$ experienced vessel perforation in $4(9.0 \%)$ of 44 patients in flow diverter stent placement and recommended consideration of change of the procedure to parent artery occlusion since marked tortuousness of the parent artery increases the risk of procedural complications including vessel perforation.

A characteristic of perforation of a distant vessel as in our patient is the difficulty in its detection during the procedure, first because the surgeon, whose attention is concentrated on the lesion area, is unlikely to notice changes in the condition of distal vessels, and second because clear extravasation is often absent during the procedure in perforation of distal vessels. Ryu et al. ${ }^{3)}$ reported that no change in blood pressure or extravasation was noted during the procedure in three 


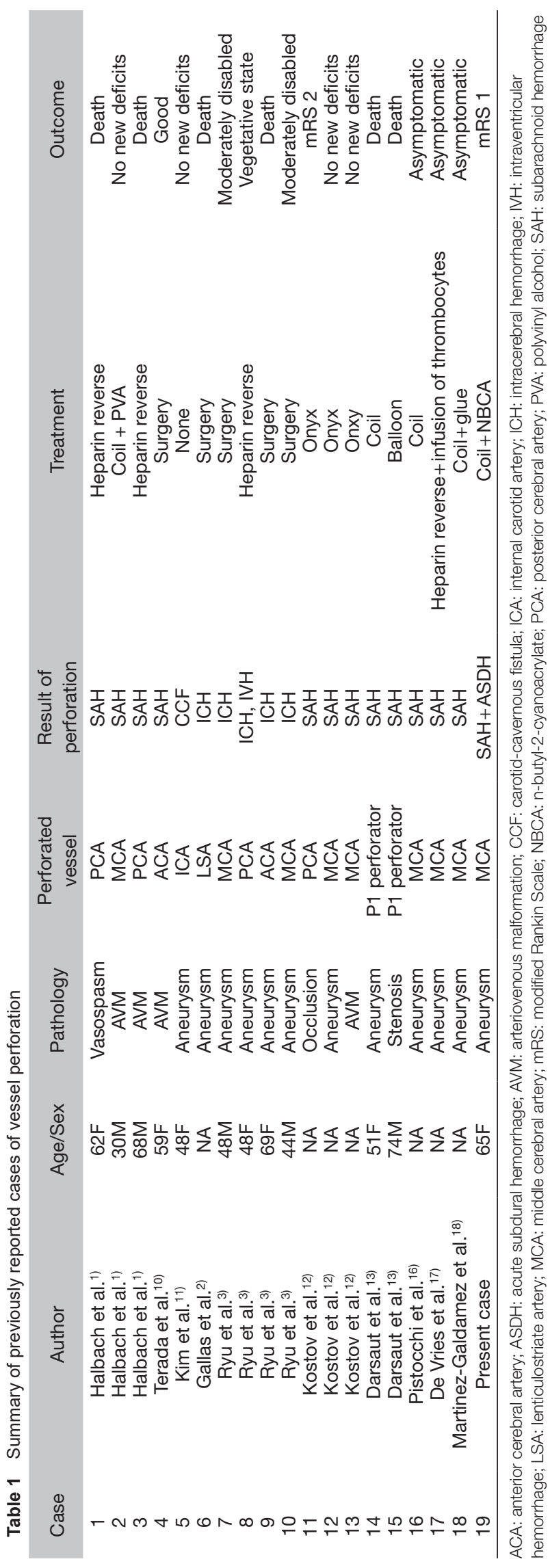

(75\%) of the four patients who underwent coil embolization of intracranial aneurysm, and the occurrence of vessel perforation was difficult to perceive. Gallas et al. ${ }^{2)}$ also reported that perforation of the lenticulostriatal artery could not be perceived during coil embolization of intracranial aneurysm in one patient and was first noticed by intracerebral hemorrhage observed on postprocedural CT. However, as a small amount of blood leakage also persists in such patients showing no extravasation, attention to the possibility of massive bleeding, which aggravates the outcome, is necessary. Other than extravasation, stagnation and prolongation of the washout time of the contrast medium and localized vasospasm have been reported as findings associated with perforation of small distal arteries. ${ }^{3)}$ In the event of detection of such findings, the possibility of vessel perforation must be considered. In addition to the above findings, checking the presence or absence of hemorrhagic complications in CT-like images obtained by cone beam $\mathrm{CT}$ at the end of the treatment is also useful for the prevention of overlooking.

\section{Treatment for perforation of distal vessels}

To stop bleeding from perforated vessels, rapid reversal of heparin anticoagulation by intravenous protamine injection is first performed in most cases. The subsequent measures vary among patients, but there have been a few reports that bleeding could be stopped by reversal of heparin anticoagulation alone when the hole was made by perforation with a wire and was small or by temporary occlusion of the perforated area by balloons. ${ }^{3,18,20)}$ However, if bleeding cannot be stopped by such less invasive measures, it is necessary to consider occlusion of the perforated area together with the parent artery. Vessel occlusion is performed using coils, liquid embolic agents (e.g., NBCA, Onyx), polyvinyl alcohol (PVA), or detachable balloons. ${ }^{1,3,18,19)}$ Furthermore, if appropriate hemostasis is judged not to be achieved by these methods, craniotomy (surgical hemostasis) may be performed. ${ }^{2,3,16)}$ Which of these treatments are superior is unclear from past reports, and case-by-case evaluation is considered necessary. In our patient, we placed a flow diverter stent, and as long-term dual antiplatelet therapy was considered necessary for the prevention of postprocedural stent thrombosis, we performed trapping of the perforated area without attempting temporary balloon occlusion.

\section{Conclusion}

We experienced perforation of a distal blood vessel caused by the delivery wire during placement of the Pipeline Flex. 
Iatrogenic vessel perforation in intracranial endovascular treatment is a very serious complication, and we realized, with remorse, the importance of awareness of risky conditions and catheter manipulations and not failing to notice even slight angiographic abnormalities for its prevention.

\section{Disclosure Statement}

Akira Ishii has received payments such as lecture fees from Medtronic (Minneapolis, MN, USA). Neither the first author nor any coauthor has any conflicts of interest.

\section{References}

1) Halbach VV, Higashida RT, Dowd CF, et al: Management of vascular perforations that occur during neurointerventional procedures. AJNR Am J Neuroradiol 1991; 12: 319-327.

2) Gallas S, Drouineau J, Gabrillargues J, et al: Feasibility, procedural morbidity and mortality, and long-term follow-up of endovascular treatment of 321 unruptured aneurysms. AJNR Am J Neuroradiol 2008; 29: 63-68.

3) Ryu CW, Lee CY, Koh JS, et al: Vascular perforation during coil embolization of an intracranial aneurysm: the incidence, mechanism, and clinical outcome. Neurointervention 2011; 6: $17-22$.

4) Lin LM, Colby GP, Jiang B, et al: Classification of cavernous internal carotid artery tortuosity: a predictor of procedural complexity in Pipeline embolization. J Neurointerv Surg 2015; 7: 628-633.

5) Baharvahdat $\mathrm{H}$, Blanc R, Termechi $\mathrm{R}$, et al: Hemorrhagic complications after endovascular treatment of cerebral arteriovenous malformations. AJNR Am J Neuroradiol 2014; 35: 978-983.

6) Murayama Y, Viñuela F, Duckwiler GR, et al: Embolization of incidental cerebral aneurysms by using the Guglielmi detachable coil system. J Neurosurg 1999; 90: 207-214.

7) Cloft HJ, Kallmes DF: Cerebral aneurysm perforations complicating therapy with Guglielmi detachable coils: a meta-analysis. AJNR Am J Neuroradiol 2002; 23: 1706-1709.

8) Park HK, Horowitz M, Jungreis C, et al: Periprocedural morbidity and mortality associated with endovascular treatment of intracranial aneurysms. AJNR Am J Neuroradiol 2005; 26: 506-514.

9) Flint AC, Duckwiler GR, Budzik RF, et al: Mechanical thrombectomy of intracranial internal carotid occlusion: pooled results of the MERCI and Multi MERCI Part I trials. Stroke 2007; 38: 1274-1280.

10) IMS Study Investigators. Combined intravenous and intraarterial recanalization for acute ischemic stroke: the interventional management of stroke study. Stroke 2004; 35: 904-911.

11) Pistocchi $S$, Blanc R, Bartolini B, et al: Flow diverters at and beyond the level of the circle of willis for the treatment of intracranial aneurysms. Stroke 2012; 43: 1032-1038.

12) De Vries J, Boogaarts J, Van Norden A, et al: New generation of flow diverter (surpass) for unruptured intracranial aneurysms: a prospective single-center study in 37 patients. Stroke 2013; 44: 1567-1577.

13) Piano M, Valvassori L, Quilici L, et al: Midterm and longterm follow-up of cerebral aneurysms treated with flow diverter devices: a single-center experience. $J$ Neurosurg 2013; 118: 408-416.

14) Martínez-Galdámez $M$, Romance $A$, Vega $P$, et al: Pipeline endovascular device for the treatment of intracranial aneurysms at the level of the circle of Willis and beyond: multicenter experience. J Neurointerv Surg 2015; 7: 816-823.

15) Puffer RC, Piano M, Lanzino G, et al: Treatment of cavernous sinus aneurysms with flow diversion: results in 44 patients. AJNR Am J Neuroradiol 2014; 35: 948-951.

16) Terada $T$, Okuno $T$, Hayashi $S$, et al: A case of vessel perforation during interventional neuroradiological procedure. Operative findings of the perforated vessel. Surg Neurol 1993; 40: 241-244.

17) Kim TS, Ezura M, Takahashi A: Guidewire-induced carotid cavernous fistula. Interv Neuroradiol 2001; 7: 161-165.

18) Kostov D, Kanaan H, Lin R, et al: Repair of intracranial vessel perforation with Onyx-18 using an exovascular retreating catheter technique. J Neurointerv Surg 2012; 4: 121-124.

19) Darsaut TE, Costalat V, Salazkin I, et al: Fatal avulsion of choroidal or perforating arteries by guidewires. Case reports, ex vivo experiments, potential mechanisms and prevention. Interv Neuroradiol 2014; 20: 251-260.

20) Sakai N, Ezura M, Matsumaru Y, et al: Progress of intracranial endovascular treatment 2015. 1st edition. Tokyo, Shindan to Chiryo Sha, 2014; 42-47. 